\title{
Numerical modelling of the lobes of radio galaxies in cluster environments - IV. Remnant radio galaxies
}

\author{
W. English ${ }^{1}$, M. J. Hardcastle ${ }^{1 \star}$ and M. G. H. Krause ${ }^{1}$ \\ ${ }^{1}$ Centre for Astrophysics Research, School of Physics, Astronomy and Mathematics, University of Hertfordshire, College Lane, Hatfield, AL10 9AB, UK
}

Accepted XXX. Received YYY; in original form ZZZ

\begin{abstract}
We examine the remnant phase of radio galaxies using three-dimensional hydrodynamical simulations of relativistic jets propagating through cluster environments. By switching the jets off once the lobes have reached a certain length we can study how the energy distribution between the lobes and shocked intra-cluster medium compares to that of an active source, as well as calculate synchrotron emission properties of the remnant sources. We see that as a result of disturbed cluster gas beginning to settle back into the initial cluster potential, streams of dense gas are pushed along the jet axis behind the remnant lobes, causing them to rise out of the cluster faster than they would due to buoyancy. This leads to increased adiabatic losses and a rapid dimming. The rapid decay of total flux density and surface brightness may explain the small number of remnant sources found in samples with a high flux density limit and may cause analytic models to overestimate the remnant fraction expected in sensitive surveys such as those now being carried out with LOFAR.
\end{abstract}

Key words: hydrodynamics - methods: numerical - galaxies: active - galaxies: jets

\section{INTRODUCTION}

The basic mechanism by which the lobes of powerful radio galaxies are powered has been understood for some time: an accreting active galactic nucleus (AGN) launches bipolar jets of relativistic plasma into the surrounding intracluster medium (ICM) (Begelman, Blandford \& Rees 1984; Bridle \& Perley 1984). In standard models (Blandford \& Rees 1974; Scheuer 1974) these jets impact the local ICM and end in a termination shock, depositing the spent jet material around the head of the jet where it goes on to inflate an overpressured cocoon of low-density plasma which will drive a shell of shocked ICM around the lobes. Eventually the lobes will come into pressure equilibrium with the local ICM, but will continue to expand while energy and momentum is constantly supplied by the central AGN (Hardcastle \& Worrall 2000; Croston et al. 2004; Hardcastle \& Krause 2013). However, the evolution of radio galaxies once the jets are switched off, known as the remnant phase, is less well understood.

A number of radio-loud AGN have been identified that appear to have no current jet activity (eg. Cordey 1987; Hardcastle et al. 1997; Gentile et al. 2007), and estimates of the time taken for the remnant source to cool radiatively below observational limits (up to $10^{8} \mathrm{Myr}$, Cordey (1986)) suggest that this timescale should be roughly similar to the active phase of the AGN, though this estimate assumes that radiative losses are dominant, whereas sources that remain overpressured up to their remnant phase are expected to dim much more quickly due to adiabatic losses (Kaiser \& Cot-

^ Corresponding author. E-mail: m.j.hardcastle@ herts.ac.uk ter 2002). Observationally these remnant objects remain rare, with only a small percentage of radio sources being classified as remnants. Giovannini et al. (1988) find that in a sample of 187 radio galaxies from the $\mathrm{B} 2$ and $3 \mathrm{C}$ catalogues, only $\approx 3$ per cent are probably remnants. By selection, samples with high flux density limits have poor sensitivity to remnants, but LOFAR observations and modelling give upper limits on the remnant fraction of $\sim 10$ per cent (Brienza et al. 2017; Mahatma et al. 2018). Although the numbers of both are small, true remnant sources may be even rarer than double-double radio galaxies (Schoenmakers et al. 2000), those in which a new active phase creates lobes clearly distinguishable from those formed in an earlier phase, despite the short timescale it should take for the new lobes to merge with the old (Clarke \& Burns 1991; Kaiser, Schoenmakers \& Röttgering 2000; Konar \& Hardcastle 2013; Konar et al. 2013; Mahatma et al. 2019). This suggests that the fading time of remnant sources is very short.

Numerical modelling of radio galaxies has until recently focused on the active phase - understandably since, as seen above, they make up the vast majority of observed sources. Due to the vastly different spatial scales needed to model radio galaxies in their entirety, from the launching of the jets to their impact on the cooling of cluster gas, simulations are forced to focus on three main areas. Large-scale cosmological simulations including AGN feedback, either explicitly or via semi-analytic post-processing, are able to reproduce observed mass and luminosity functions of galaxies (Croton et al. 2005; Bower et al. 2006); however, the jets are not directly modelled as this would be computationally infeasible, and instead feedback is included by directly giving the affected particles an amount of energy (usually either as thermal or a combination of 
thermal and kinetic) dependent on values such as the black hole's mass and an observationally determined feedback efficiency (eg. Vogelsberger et al. 2014; Schaye et al. 2015). At the other end of the scale are models which simulate the black hole and its immediate surroundings, out to a few hundred Schwarzschild radii, which can allow the investigation of the efficiency of the jet launching process as well as the energy content of the jets (eg. Tchekhovskoy, Narayan \& McKinney 2011). In between these are those models which inject an already formed and collimated jet into some form of environment; from a simple uniform distribution (Norman et al. 1982; Lind et al. 1989) to a more realistic beta model (Basson \& Alexander 2003; Krause 2005), and even using an environment derived from simulations of a dynamically active cluster (Heinz et al. 2006; Bourne \& Sijacki 2017). Studies of the evolution of radio lobes that have covered the remnant phase are mostly of the last type (e.g. Basson \& Alexander 2003; Zanni et al. 2005; Perucho et al. 2011, 2014) and, to date, have concentrated on the dynamics of the remnant sources and their effect on the external medium rather than on their observational properties.

In previous work (Hardcastle \& Krause 2013, 2014; English, Hardcastle \& Krause 2016, hereafter Papers 1, 2 and 3) we investigated the impact of an active jet, powered by an AGN, on the surrounding ICM by way of numerical modelling using the last of the approaches described above. Paper 1 used two-dimensional, purely hydrodynamical (HD) models of AGN jets in a range of environments to study the resulting radio lobes. Paper 2 extended this to three-dimensional magnetohydrodynamical (MHD) models, allowing synthetic synchrotron observations to be created and the observational properties to be studied in varying environments. Paper 3 took this a step further and used relativistic MHD (RMHD) models to test the effects of jet power and velocity on the lobe dynamics and energetics. Here we present results from a set of threedimensional RMHD models in which the jet is switched off while the lobes are still evolving in the cluster environment. The inclusion of both relativity and magnetic fields in our models allows us to simulate observations of remnant radio sources, to investigate their impact on the external medium and to and make predictions for their observability in different radio surveys. In Section 2 we will describe how the simulations are set up and list the parameters used for the jet and cluster. In Section 3 we present and discuss the results from this set of simulations, and Section 4 summarizes our findings.

\section{SIMULATION SETUP}

We make use of the freely available code PLUTO $^{1}$, version 4.2, described by Mignone et al. (2007). The models are performed using the RMHD physics module, the HLLD approximate Riemann solver, and second order dimensionally unsplit Runge-Kutta time stepping algorithm with a Courant-Friedrichs-Lewy number of 0.3 . We use the Taub-Matthews equation of state which has a variable adiabatic index which varies with temperature, ranging from the low temperature (ideal gas) value of $5 / 3$ to the high temperature (relativistic plasma) value of $4 / 3$. A divergence cleaning algorithm is used to enforce $\nabla \cdot \mathbf{B}=0$. We do not use the adaptive mesh refinement (AMR) capability of PLUTO.

For consistency with our previous work we define simulation units of length, $L_{0}$, and density, $\rho_{0}$, to be $2.1 \mathrm{kpc}$ and $3.011 \times 10^{-23}$

${ }^{1}$ http://plutocode.ph.unito.it $\mathrm{kg} \mathrm{m}^{-3}$ (giving a unit number density, $n_{0}$, of $3 \times 10^{4} \mathrm{~m}^{-3}$, for a mean particle mass $\mu$ of $0.6 m_{p}$ ); the simulation units for velocity must be the speed of light, $c$, for the RMHD module. From these the remaining units can be derived, giving simulation units for time $\left(t_{0}=L_{0} / v_{0}\right)$ to be $6.85 \mathrm{kyr}$, magnetic field strength $\left(B_{0}=v_{0} \sqrt{4 \pi \rho_{0}}\right)$ to be $1.84 \mu \mathrm{T}$, and pressure $\left(p_{0}=\rho_{0} v_{0}^{2}\right)$ to be $2.7 \times 10^{-6} \mathrm{~Pa}$. As in Paper 3, we set the central pressure in the cluster $p_{0}$ to be $10^{-10} \mathrm{~Pa}$ in physical units, or $\left(2.7 \times 10^{4}\right)^{-1}$ in simulation units.

We simulate a 400 by 400 by 400 element volume ranging between $\pm 150 L_{0}$, giving a physical resolution of $1.6 \mathrm{kpc}$ and allowing each lobe to expand to a length of $315 \mathrm{kpc}$. The grid has periodic outer boundary conditions (in order to enforce zero velocity at the boundary), and a cylindrical internal boundary condition is defined at the centre of the grid, aligned with the $x$-axis, from which the jet is injected. This internal region has radius $r_{j}=2 L_{0}$, giving a jet resolution of 2.7 cells per jet radius, and length $l_{j}=3 L_{0}$. While this gives an unphysically large jet radius $\left(r_{j}=4.2 \mathrm{kpc}\right)$ we are limited by the numerical resolution, as the jet has to have a high enough resolution for the internal boundary to couple well to the local environment. We are more concerned about the physics of the lobes than those of the jet, and we found, in Paper 2 and Paper 3 , that these models with a relatively low resolution across the jet were sufficient to capture the lobe physics well, as demonstrated by the agreement between Paper 2 and Paper 3 and other, higherresolution, models for properties such as the evolution of the lobes' volume and axial ratio, so in general we are not concerned by this feature of the models. It is, however, worth noting that the low resolution across the jet could lead to numerical diffusion, preventing turbulence and instabilities which are key in amplifying the injected magnetic field.

Inside the injection region we define the density $\rho_{j}$, temperature $T_{j}$, velocity $\mathbf{v}_{j}= \pm \mathbf{i} v_{x}\left(v_{y}=v_{z}=0\right)$ and magnetic field strength $\mathbf{B}_{j}$ of the injected material such that the jet is light compared to the environment, using the values shown in Table 1. A conserved tracer quantity is injected with the jet material, and initially has a value of 1.0 inside the injection region and 0 everywhere else. This is used in post-processing to distinguish between the lobes and shocked cluster material, using the same process as in Paper 3. The shocked ICM is identified by searching inwards from each edge of the grid for the surface at which the radial velocity is greater than $2.5 \times 10^{-4} c$. Similarly, for the lobes, we search inwards for the surface at which the tracer is greater than $10^{-3}$, though in the current paper we also search along the jet axis from the centre outwards, with the same criteria, to ensure that any ICM pulled up behind the lobes is not counted as lobe material.

Since we set the internal boundary flag to true inside the injection region, these cells are not evolved as part of the hydrodynamics solver. This results in the internal boundary region acting as a sink into which material and energy can be lost. While the jet is active this is not an issue, since the material injected by the jet forces the ICM away from the cluster centre. However once the jet has been turned off, or even once the lobes have started to rise out from the core, the ICM will start to relax back into the potential, falling in towards the internal boundary. To prevent this, once the lobes have reached the desired length the jets are turned off by disabling the internal boundary condition and the resulting hole is filled with stationary material that has density and pressure equal to the average values found around the outside of the injection region.

The surrounding environment used for these models is given 
by an isothermal beta model, representing that of a rich group or cluster, with density profile:

$n=n_{0}\left[1+\left(\frac{r}{r_{c}}\right)^{2}\right]^{-\frac{3 \beta}{2}}$

where $r_{c}$ is the core radius. In order to break the symmetry between the two lobes we introduce small random perturbations in the density, so that for each set of input parameters we can get results from two non-identical lobes. To keep the cluster environment stable a gravitational acceleration vector is defined by:

$\mathbf{g}=-\frac{3 \beta}{\Gamma \times 2.7 \times 10^{4}} \frac{\mathbf{r} /|\mathbf{r}|}{\sqrt{\mathbf{r}^{2}+r_{c}^{2}}}$

where the factor of $2.7 \times 10^{4}$ comes from the temperature of the cluster in simulation units. The magnetic field in the cluster is set as a Gaussian random field that has an energy density that scales with thermal pressure, described by Murgia et al. (2004) and Hardcastle (2013). This is done by generating the Fourier transform of the magnetic vector potential $\mathbf{A}(k)$. For each of the three components we draw the complex phase from a uniform distribution and the magnitude from a Rayleigh distribution whose controlling parameter depends on $k$. From this we can calculate the Fourier transform of the magnetic field, and by taking the inverse Fourier transform we obtain a divergence-free magnetic field with, after scaling to physical units, a peak field strength at the centre of the cluster of $0.7 \mathrm{nT}$.

Two sets of simulations are performed in order to study the behaviour of the lobes once they are no longer being fed by the jet. Simulation parameters for each model are presented in Table 1.

(i) The main set consists of 9 simulations in which we vary the $\beta$ and $r_{c}$ parameters of the environment in the ranges $\beta=$ $0.55,0.75,0.95$ and $r_{c}=40,60,80 \mathrm{kpc}$. The jets in each of these models are identical, with the same parameters as the v95-med-m model from Paper 3; they are powerful $\left(Q=2 \times 10^{39} \mathrm{~W}\right)$, highly relativistic $\left(v_{j e t}=0.95, \gamma_{j}=3.2\right)$, under-dense $\left(\eta=3.478 \times 10^{-6}\right)$ and include a toroidal magnetic field around the $x$-axis, where $B_{j}=0.209 \mathrm{nT}$, with $B_{y}=B_{j}(z / r)$ and $B_{z}=B_{j}(y / r)$ for $r<r_{j}$. Here $\eta$ is the relativistic generalization of the jet density contrast with the central density of the ambient medium $\rho_{0}$, as described in Paper 3. We allow the models to evolve until the lobes have reached a length of $150 \mathrm{kpc}$, at which time the jet is shut off as described above. This makes sure that the lobes have sufficient time to form while the jet is active, while ensuring enough time to study the remnant source before it reaches the edge of the simulated grid, at which time the models were terminated.

(ii) In addition, we run an additional pair of simulations, with the same cluster and jet properties as the r75-60 model, but in which the jets are turned off once they reach 110 and $190 \mathrm{kpc}$. This allows us to test how the position of the lobes within the cluster affects the time taken for the remnant to cool below an observable limit. Lobes that are overpressured compared to the local environment are expected to dim faster than those closer to pressure equilibrium, due to the increased adiabatic expansion losses.

(iii) To cover the full range of input parameters a final pair of simulations in which the jet power is varied. The first takes the same jet parameters as the v95-low-m model of Paper 3; with jet power $Q=1 \times 10^{39} \mathrm{~W}$, velocity $v_{j e t}=0.95$, density $\eta=1.739 \times 10^{-6}$ and magnetic field strength $B_{j}=0.158 \mathrm{nT}$. The second takes the parameters of v95-high-m; jet power $Q=5 \times 10^{39} \mathrm{~W}$, velocity
Table 1. Key simulation parameters for each model discussed in this paper. From left to right the columns give the code used to identify each model, the injected jet power $Q, \beta$ value and core radius $r_{c}$ of the cluster environment, and the length $L_{\text {switch-off }}$ the lobes are allowed to reach before the jet is switched off.

\begin{tabular}{ccccc}
\hline Code & $\begin{array}{c}Q \\
(\mathrm{~W})\end{array}$ & $\beta$ & $\begin{array}{c}r_{c} \\
(\mathrm{kpc})\end{array}$ & $\begin{array}{c}L_{\text {switch-off }} \\
(\mathrm{kpc})\end{array}$ \\
\hline r55-40 & $2 \times 10^{39}$ & 0.55 & 40 & 150 \\
r55-60 & & & 60 & \\
r55-80 & & & 80 & \\
r75-40 & & 0.75 & 40 & \\
r75-60 & & & 60 & \\
r75-80 & & & 80 & \\
r95-40 & & 0.95 & 40 & \\
r95-60 & & & 60 & \\
r95-80 & & 0.75 & 60 & 110 \\
r75-60-Early & & & & 190 \\
r75-60-Late & & & \\
r75-60-Low & $1 \times 10^{39}$ & & & \\
r75-60-High & $5 \times 10^{39}$ & & & \\
\hline
\end{tabular}

$v_{j e t}=0.95$, density $\eta=8.696 \times 10^{-6}$ and magnetic field strength $B_{j}=0.331 \mathrm{nT}$.

As in our earlier work, all of these models were run on the University of Hertfordshire high-performance computing facility ${ }^{2}$. 192 Xeon-based cores were used for each job with the Message Passing Interface (MPI) being implemented in MVAPICH2. Each model took around 1 week to run. Calculations are performed in post-processing from output files produced every 150 simulation time units, or every $1.02 \mathrm{Myr}$, consisting of density, velocity, pressure, magnetic field strength and tracer quantity values for the entire simulation grid. From these the energetics and dynamics of the lobes and shocked ICM are determined. We calculate values for each lobe independently due to the slight density fluctuations in the environment. Therefore lobe properties presented will be the average value for the two lobes.

\section{RESULTS AND DISCUSSION}

From here, unless otherwise stated, results will be in physical units. When discussing the dynamics of the lobes we will give results as the average value across the two lobes in each model, but for the observational properties results will be for the entire source. Where we plot results for a single model r75-60 will be used, as this is the most typical representative for the suite of models.

\subsection{Lobe dynamics and energetics}

We begin by discussing the lobe dynamics during both the active and remnant phases. Figure 1 shows snapshot midplane density and pressure slices for a number of timesteps for the r75-60 model, showing the evolution of the lobes as they are inflated by the jet and later buoyantly rise out of the centre of the cluster. The black square visible in the first two pressure maps is the injection region, which is turned off and filled in once the lobes reach a length of 150 $\mathrm{kpc}$ for the main suite of models, which occurs at 50 Myrs here.

2 https://uhhpc.herts.ac.uk/ 
We can see that while the jet is active the lobes are visible against the surrounding cocoon of shocked ICM in the pressure maps, as during this phase they are still overpressured with respect to their environment. Once the jets are switched off they very quickly reach pressure equilibrium. Switching off the jet also has a large effect on the shape of the shock driven through the cluster; as there is no longer a much stronger force pushing the shock in the longitudinal direction, it quickly takes on a more spherical shape. In the density maps we see that as the unpowered lobes are floating outwards they do not keep their prolate spheroidal shape, but instead take on more of a ' $U$ ' shape as some of the dense cluster material being dragged along by the jet pushes inside the back of the remnant lobe and hollows it out. This can be better seen in the left side of Figure 2, which shows midplane slices of momentum density for the same timesteps for the r75-60 model. Here we clearly see in the last two plots that a large amount of dense cluster gas is falling back into the potential and refilling the cluster's initial density distribution, but the weight of this infalling gas is also pushing 'streams' of material along the channel left by the jets and into the lobes. For some models this dense gas has pushed all the way through to the front of the lobes, breaking them apart, by the end of the simulations. Combined with the fact that the lobes are still overpressured at their tips after the switch-off of the jet, this has the effect of pushing the lobes out faster than they would buoyantly rise (as demonstrated in Figure 3), driving them out to lower pressure regions of the cluster where they will undergo faster adiabatic expansion, and in some cases destroying the lobes before they reach the edge of the grid.

The right hand side of Figure 2 shows the evolution of the magnetic field strength. While the jets are active the majority of the magnetic energy density is located in the lobes, as they are constantly being fed by the injected toroidal field. Also visible is the very strong field that forms around the edge of the lobes, caused by the compression of the initial cluster field by the shock. After a short while in the remnant phase the magnetic energy density in the lobes drops significantly, due to the expansion of the lobes with no new energy being added. Instead the majority of the magnetic energy now comes from the compressed and shear-amplified cluster field which has been pulled around the back of the lobes and is settling back in the centre of the cluster.

The dynamics of the lobes (Fig. 4) are, as expected, the same as in Paper 2 and Paper 3 during the active phase for the different environments. In models with a smaller core radius the lobes grow faster both in terms of length and volume, as they quickly leave the dense core and push out into the lower-pressure outer cluster. Similarly, environments with a steeper density gradient (higher values of $\beta$ ) lead to faster lobe growth at late times. Once the jets are turned off the lobes quickly slow their growth, with environment having little effect on the growth of the lobes: all of the models settle into the same expansion speed.

Figure 5 shows the evolution of the different forms of energy with time for the r75-60 model. Due to difficulty getting the internal boundary and the external environment to couple well at early times, the total injected energy is lower than the expected value until the lobes have formed and the shock has evacuated the material immediately surrounding the injection region. Since the fraction of energy being injected in each of the different components stays constant during this time, and since the focus of this paper is on the remnant phase, this early expansion should have little effect on our results. The cluster's energy content, however, undergoes a significant change during the remnant phase. As soon as the jet is no longer actively driving the shock the temperature of this thermal gas drops and it begins falling back down the cluster potential. We can also see that immediately after the jets switch off the total energy stored in the lobes drops significantly, as they continue to expand and do work on the ICM but without their own supply of energy. As the lobes approach pressure equilibrium with the cluster gas, however, the rate at which they transfer energy to the ICM slows. This is also seen in Figure 6, which plots the ratio of energy stored in the cluster to that in the lobes. As in the previous models with a relativistic equation of state we find that the ratio of energy for an active well-formed lobe is in the range 1.5 to 2 , with environments with a smaller core radius and steeper density gradient leading to a higher percentage of the energy going into the cluster. Once the jet is switched off the ratio rapidly increases; interestingly, this occurs at roughly the same rate regardless of cluster properties or even the lobes' position within the cluster once the jet is switched off. Part of the reason for this transfer could in principle be mixing between the lobe and cluster material, and the way in which we identify the two regions: if sufficient mixing occurs to drop the value of the tracer in a cell below our threshold the cell will no longer be flagged as part of the lobe, and that energy will be counted towards the cluster's total instead. However, we believe that the transfer of energy is in fact is a genuine consequence of the altered lobe dynamics in the remnant phase. Overall mixing is seen to have only a minor effect on the amount of energy stored in each region, with the calculated ratio being consistently within the range 1.5 to 2 when the value of the tracer threshold is varied. The bulk of the energetic effect seems to be due to the bulk increase of the potential and kinetic energy of the shocked material at the expense of the lobes. Similar effects are seen in other simulations of the same dynamical situation (Perucho et al. 2011).

The expansion of remnant radio lobes has a broad similarity to a Sedov explosion and can be qualitatively understood by considering the energetics of a thin-shell model (Krause 2003; Krause \& Diehl 2014): a blast wave driven by a time-dependent energy input $E(t)=\mathcal{L} t^{d}$, where $\mathcal{L}$ is some dimensionally appropriate normalizing factor, in a spherically symmetric environment with density $\rho(r) \propto r^{k}$ has a kinetic energy fraction of $\epsilon_{\mathrm{k}}=\frac{d+2}{(d+1)(\kappa+5)}$. The kinetic energy is, in this approximation, in the shell, and so $r_{\mathrm{SL}}=\epsilon_{\mathrm{k}} /\left(1-\epsilon_{\mathrm{k}}\right)$ can be taken as a proxy for our ratio between energy in shock and lobes. First neglecting gravity, $d=0$ and $\kappa=-2.25$, as appropriate for a remnant in our setup, leads to $r_{\mathrm{SL}}=2.7$. In our simulations, energy is however continuously lost to gravitational potential energy, which is not taken into account in the standard thinshell blastwave solution. We can, however, take this energy loss of the blastwave system to gravitational potential energy into account qualitatively, by using a negative $d$. This leads to a further increase of $r_{\mathrm{SL}}$ in qualitative agreement with our findings.

\subsection{Observational properties}

Using the same process as in Paper 3 we calculate the synchrotron emissivities for the different Stokes parameters for each cell in the simulation grid. Since the emission is anisotropic this must be repeated across a range of viewing angles, achieved by defining a projection vector pointing from the centre of the grid to the observer. The synchrotron emission for a given cell is then calculated for a transformed version of this projection vector to take into account relativistic aberration, where the apparent position of an observer in the reference frame of an object moving at relativistic speeds differs to the position of the observer in the lab frame. From this we cal- 


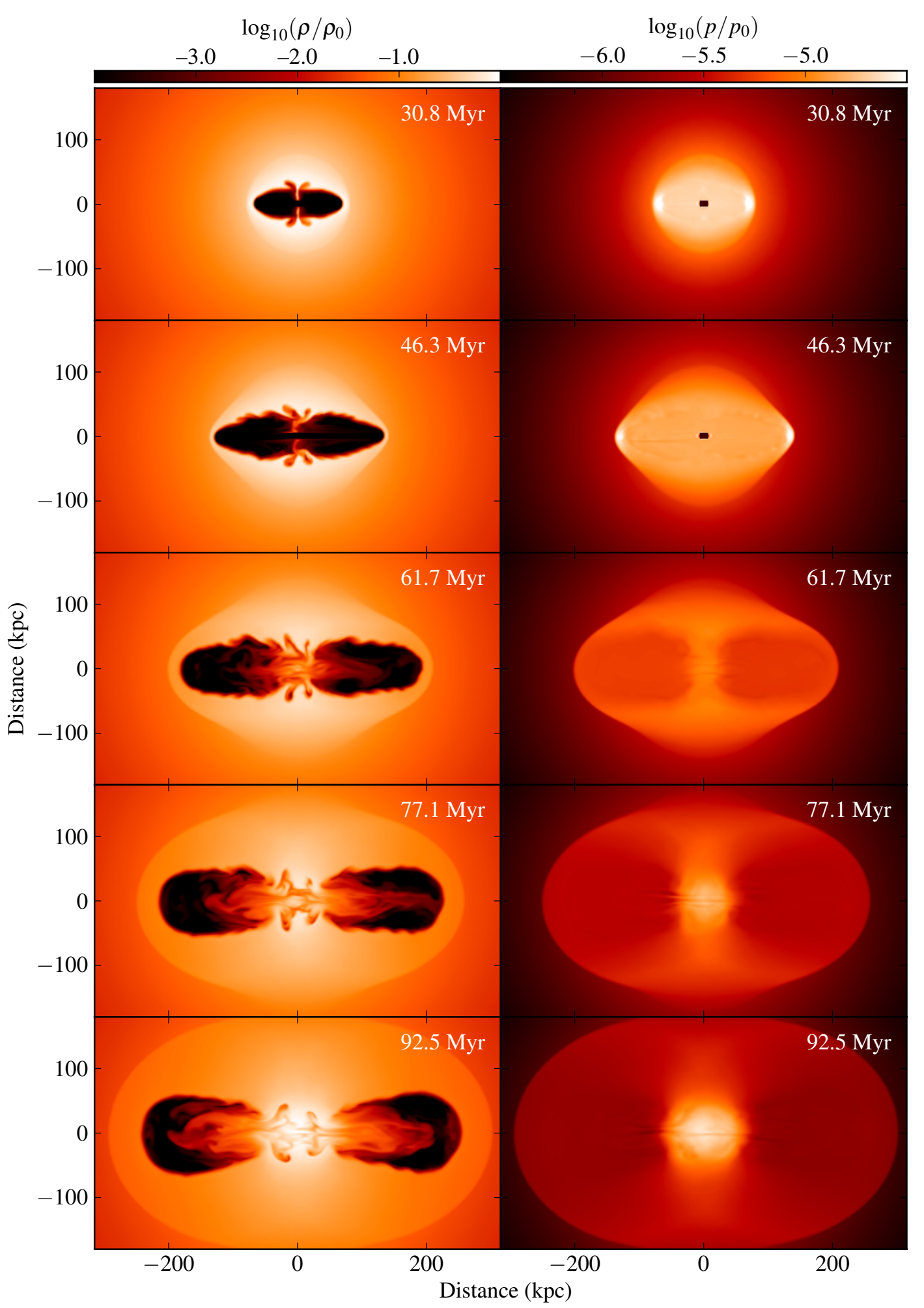

Figure 1. Midplane density and thermal pressure slices for the r75-60 model at 5 different timesteps. Colour scales are logarithmic in simulation units of density and pressure, ranging from -4.0 to 0.0 in density and from -6.5 to -4.5 in pressure. The jet is switched off once the lobe reaches $150 \mathrm{kpc}$, which in this model occurs at 50 Myr. Timestamps give the age of the model at each step. Not included in these plots is the contribution to the pressure from the magnetic field, which accounts for the slight underpressure of the lobes that appears in these plots. 


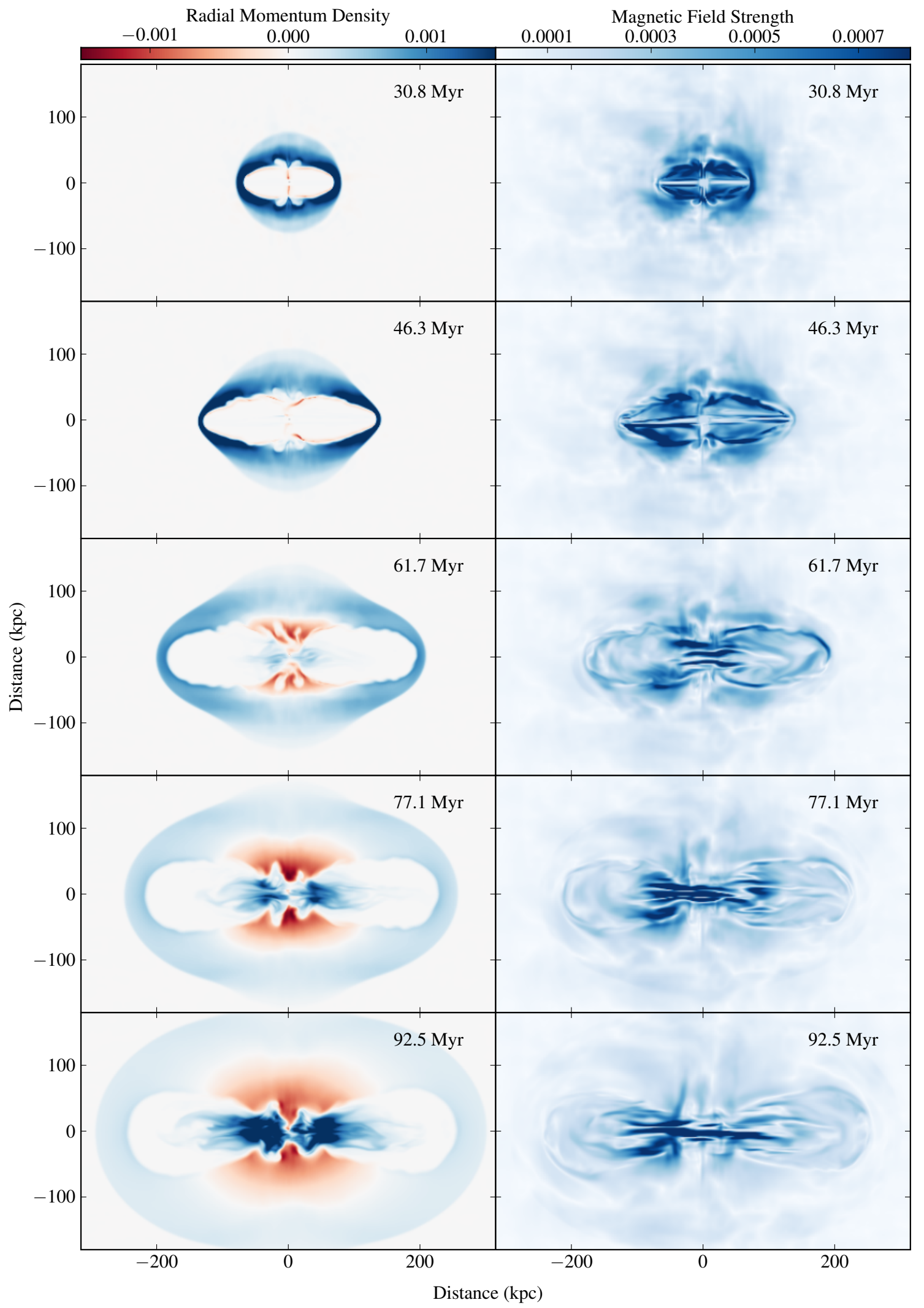

Figure 2. Midplane magnitude of the radial momentum density and magnetic field strength slices for the r75-60 model, at the same timesteps as Figure 1. Colour scales are linear in momentum and magnetic field strength, both in simulation units, ranging from -0.0015 to 0.0015 in momentum density and from 0.0 to 0.0008 in magnetic field strength. 


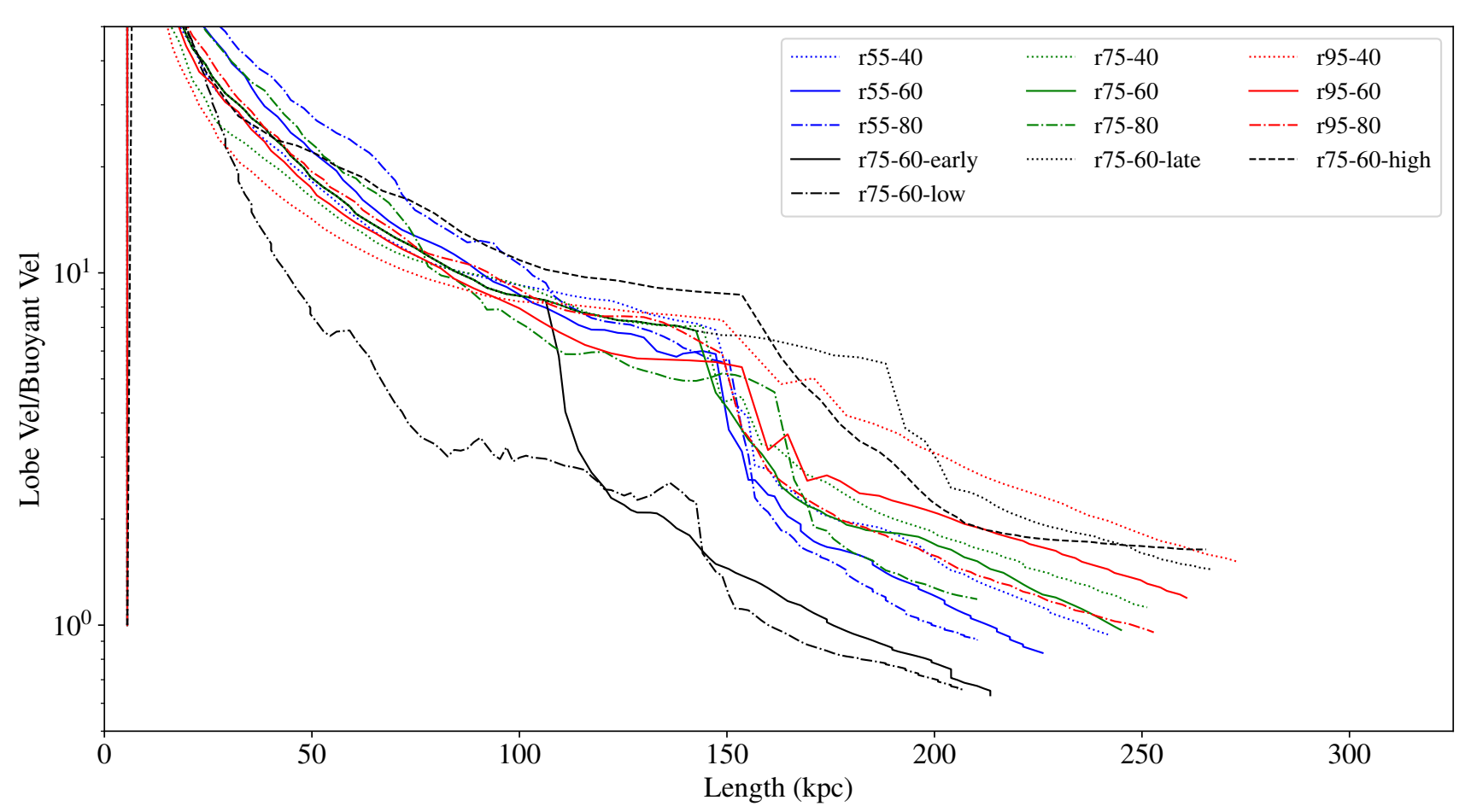

Figure 3. Ratio of the advance velocity of the lobes in each model to the velocity of a bubble of low density gas, at the same position within the cluster as the lobes, moving purely due to buoyancy showing that for a significant amount of time after the jets have been switched off the lobes continue to be forced out of the cluster by more than just buoyancy.

culate the Stokes $I$ (total intensity), $Q$ and $U$ (polarized intensities) parameters (in simulation units) using the following equations:

$j_{I}=p\left(B_{x}^{2}+B_{y}^{2}\right)^{\frac{\alpha-1}{2}}\left(B_{x}^{2}+B_{y}^{2}\right) D^{3+\alpha}$

$j_{Q}=\mu p\left(B_{x}^{2}+B_{y}^{2}\right)^{\frac{\alpha-1}{2}}\left(B_{x}^{2}-B_{y}^{2}\right) D^{3+\alpha}$

$j_{U}=\mu p\left(B_{x}^{2}+B_{y}^{2}\right)^{\frac{\alpha-1}{2}}\left(2 B_{x} B_{y}\right) D^{3+\alpha}$

where, for a fixed power-law electron energy distribution, the local thermal pressure $p$ is proportional to the electron number density. $\alpha$ is the power-law synchrotron spectral index (taken to be $\alpha=0.5$ ) and $\mu$ is the maximum fractional polarization (equal to $\mu=0.69$ for $\alpha=0.5$ ). $D$ is the Doppler factor, given by:

$D=\frac{1}{\gamma(1-\beta \cos (\theta))}$

where $\beta=v / c$ and $\theta$ is the angle between the projection vector and the velocity vector of the cell. This factor is then raised to the power $(3+\alpha)$ when calculating the emissivities to account for the increased rate at which photons are received in the lab frame compared to the rate they are emitted, the boosting of these photons to higher energies and the fact that the emitted radiation is preferentially beamed towards the direction of motion. To convert this into a more useful, physical, unit we again use a modified form of the equation from Paper 1:

$j_{0}=c(q) \frac{e^{3}}{\epsilon_{0} c m_{e}}\left(\frac{v m_{e}^{3} c^{4}}{e}\right)^{-\frac{q-1}{2}} \frac{3 p_{0}}{4 \pi I}\left(\frac{B_{0}^{2}}{8 \pi \mu_{0}}\right)^{\frac{q+1}{4}} L_{0}^{3}$ where $c(q)$ is a dimensionless constant $(\approx 0.05), e$ and $m_{e}$ are the charge and mass of an electron, $\epsilon_{0}$ and $\mu_{0}$ are the permittivity and permeability of free space and $c$ is the speed of light. For spectral index $\alpha=0.5$ the electron energy power-law index $q$ is $2, v$ is the frequency at which we observe the source and $p_{0}, L_{0}$ and $B_{0}$ are simulation units of pressure, length and magnetic field strength, respectively. Finally $I$ is the integral over $E N(E)$ between $E_{\min }$ and $E_{\max }$, with $E_{\min }=10 m_{e} c^{2}$ and $E_{\max }=10^{5} m_{e} c^{2}$. From this we get the simulation unit of radio luminosity to be $j_{0}=3.133 \times 10^{31} \mathrm{~W}$ $\mathrm{Hz}^{-1} \mathrm{sr}^{-1}$ at $150 \mathrm{MHz}$.

To allow us to compare these results with observations, such as those of Hardcastle et al. (2016), we convert these values into units of Jy. To do this we assume a standard flat $\Lambda \mathrm{CDM}$ cosmology, where $H_{0}=70 \mathrm{~km} \mathrm{~s}^{-1}, \Omega_{m}=0.3$ and $\Omega_{\Lambda}=0.7$. We place the models at redshift $z=0.6$, a fairly typical value for the AGN identified by Hardcastle et al. (2016). Using this we obtain the simulation unit of radio flux density, $f_{0}=9.185 \times 10^{5} \mathrm{Jy}$ at 150 $\mathrm{MHz}$. Results are then compared to the 3CRR flux density limit of $10 \mathrm{Jy}$ and to a surface brightness limit of $300 \mu \mathrm{Jy} \mathrm{beam}^{-1}$ for a 6 -arcsec Gaussian restoring beam, matched to the properties of the LOFAR Two-Metre Sky Survey, LoTSS (Shimwell et al. 2017, 2019). We include the effects of cooling due to synchrotron and inverse-Compton emission using the code from Hardcastle (2018), which calculates a time-dependent correction factor that is applied to our synchrotron emissivities assuming that a population of electrons in a power-law distribution is supplied at a constant rate while the jet is active, and uses the average magnetic field strength history in the lobes. These cooling processes have a significant effect on the calculated luminosities of our models (Figure 7); for a model at a redshift of $z=0.6$ the luminosity has dropped by around an order of magnitude more when cooling is included, mostly due to 

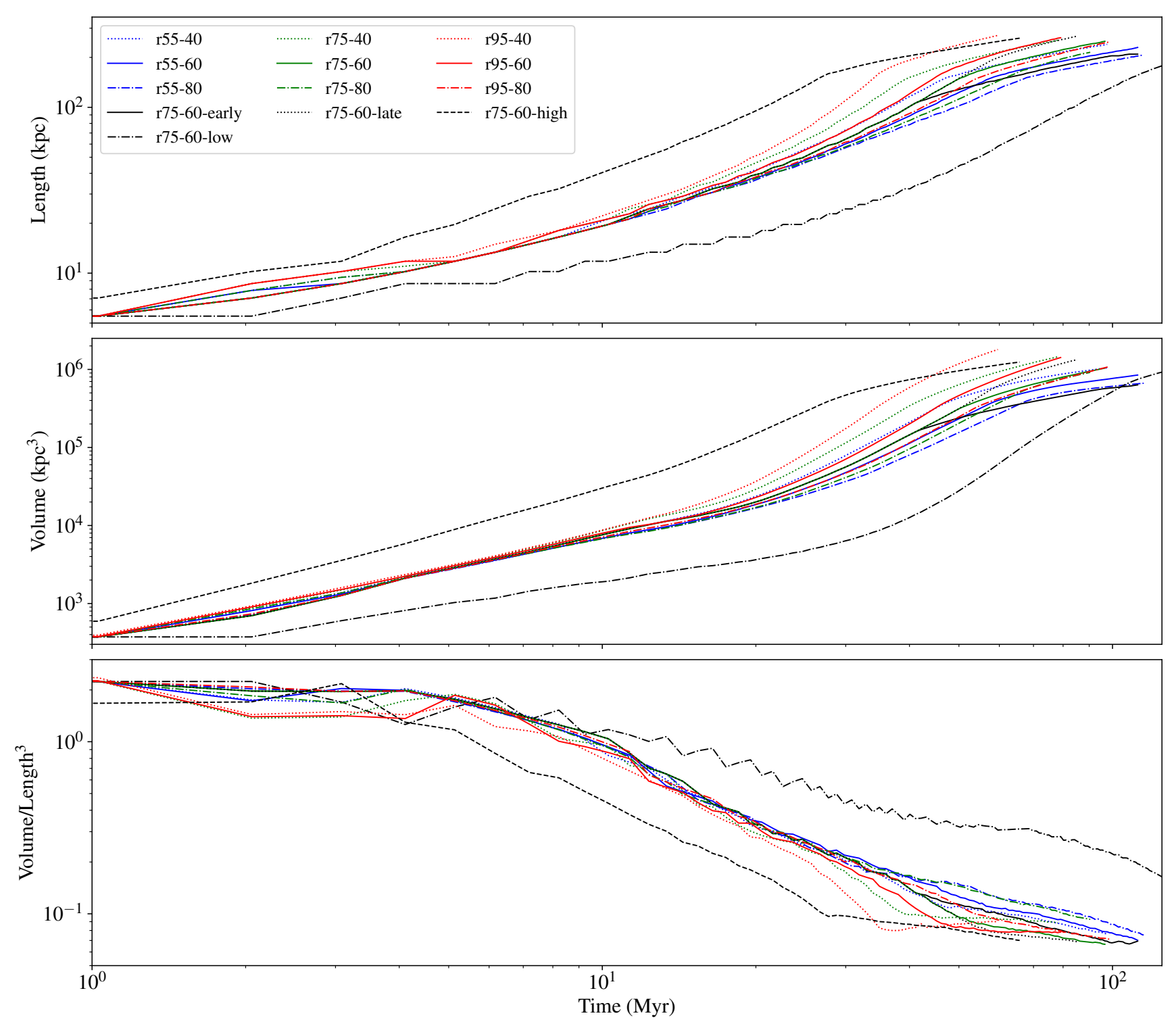

Figure 4. Growth of the lobes with time for all of the simulations run in this paper, in terms of the lobes' length (top), volume (middle) and axial ratio (bottom). For each model the plotted value is the average value for the two lobes.

the inverse-Compton losses. However, relative to the analytic models of Hardcastle (2018), the uncorrected synchrotron luminosity drops more rapidly once the jet is switched off. We attribute this to the effects of the returning cluster-centre gas on the dynamics, as discussed in Section 3.1, since the continued pressure-driven expansion of the remnant lobes is accounted for in the analytical models.

In Figure 8 we show the synchrotron light curves for each of the models, plotted in terms of rest-frame $150-\mathrm{MHz}$ flux density at $z=0.6$. The expected trends are again repeated here while the jet is active: environments with a larger core radius lead to brighter sources while the steepness of the environment has little effect, with shallower slopes possibly leading to higher luminosities. Once the jets are switched off the luminosity drops rapidly for all of the models, falling by around an order of magnitude by the time the lobes have grown by a further $50 \mathrm{kpc}$. The rate at which the luminosity decays is very similar for each model, with the main difference be- ing for the models where $\beta=0.55$, which after the initial drop all start to flatten out more noticeably than the other models. We see that for these powerful sources in rich environments, as expected, the total flux density at the peak of activity would put them in the category of 3C sources (except for r75-60-low). However, within a few Myr of the cessation of jet activity, their total flux density drops below the $3 \mathrm{C}$ limit. This behaviour may go some way towards explaining the very low fraction of candidate remnant sources seen in catalogues selected with a high flux limit (Section 1).

To get a better idea for how quickly the remnant source fades we look at how the mean surface brightness of each models evolves with time (Figure 9). From this plot there is a clear dependence on both environment properties, in the sense that flatter density profiles and larger core radii imply lower surface brightness gradients. A steeper declining density and pressure gradient means that by the time the jets are switched off, which occurs once the lobes reach a fixed length of $150 \mathrm{kpc}$, the lobes will be contained within lower- 


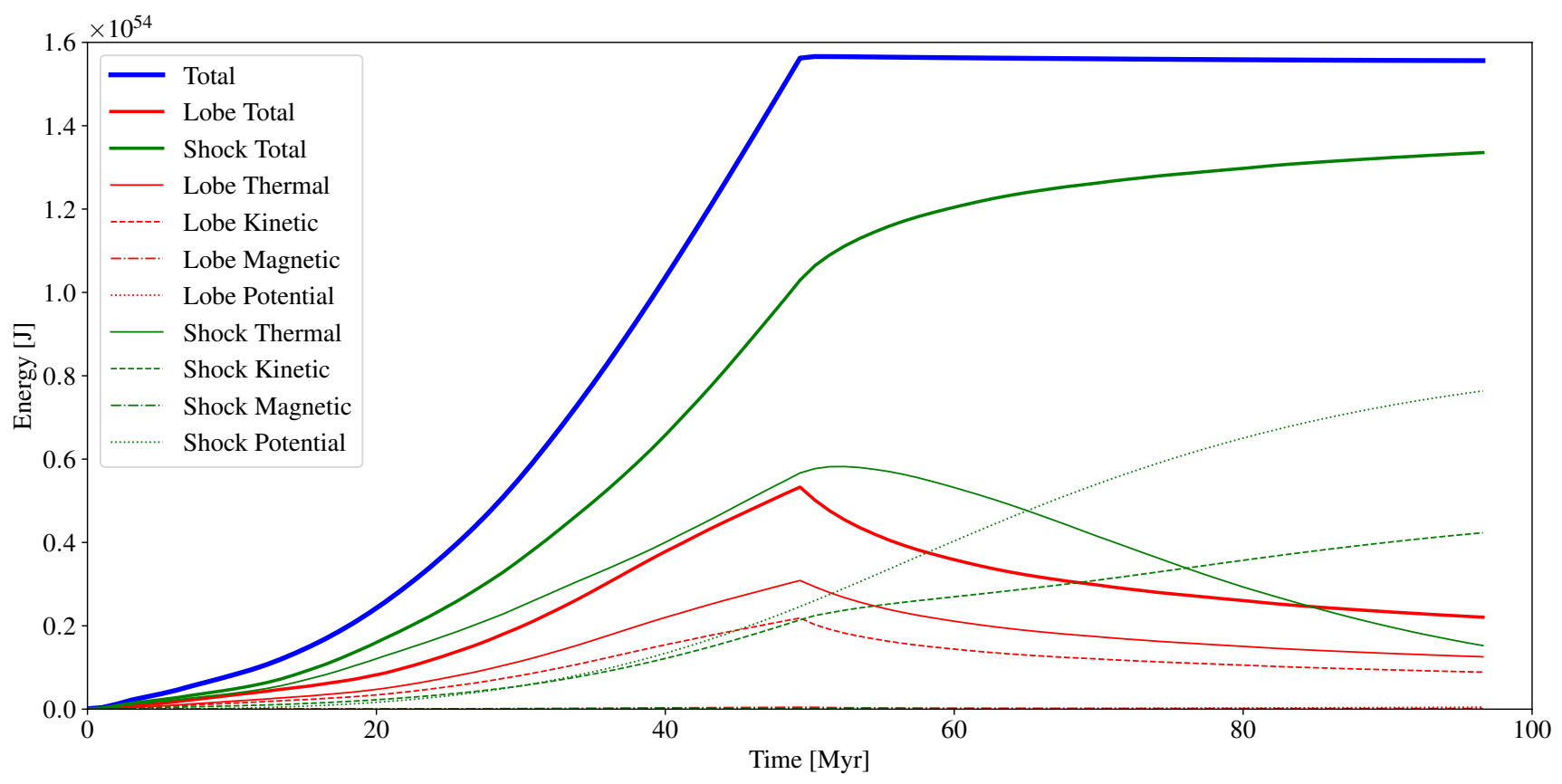

Figure 5. Evolution of the energy stored in the lobes and shocked cluster regions for the r75-60 model. As in previous models the internal boundary does not couple well with the external environment for some time, resulting in less than the intended injected energy making it onto the grid until the lobes are reasonably well formed. Once the jet is switched off, at $t \approx 50 \mathrm{Myr}$, energy is lost from the lobes and gained by the shocked medium.

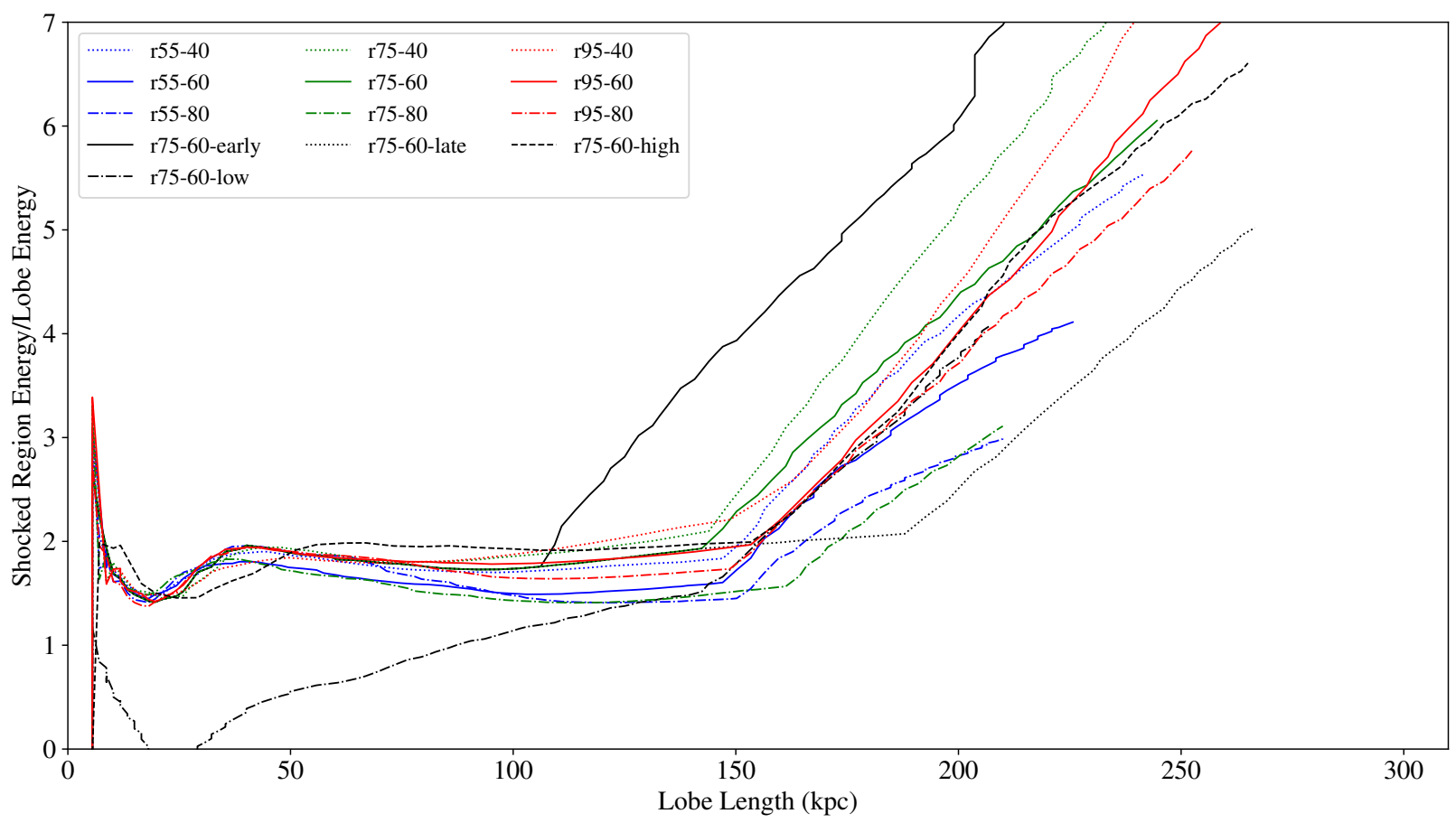

Figure 6. Ratio of energy stored in the shocked ICM to that stored in the lobes for all of the models, as a function of lobe length. The sharp increase in the ratio (at lengths $>150 \mathrm{kpc}$ in most models) takes place after the jet is switched off.

pressure cluster material and so will undergo a faster stage of adiabatic expansion than those models that are within a denser environment. During this adiabatic expansion the surface brightness drops rapidly as both the magnetic field strength and the particle energies decrease (eg. Kaiser \& Cotter 2002). Similarly we see that a large core radius has the opposite effect. This is essentially for the same reason: the larger the core radius the closer to pressure equilibrium the lobes will be once the jets are turned off, therefore the expansion of these lobes in the remnant phase is expected to be much more relaxed. Further evidence for this comes from the two models in which the jets were switched off earlier and later. While we had expected that the earlier model (r75-60-early) would still be highly 


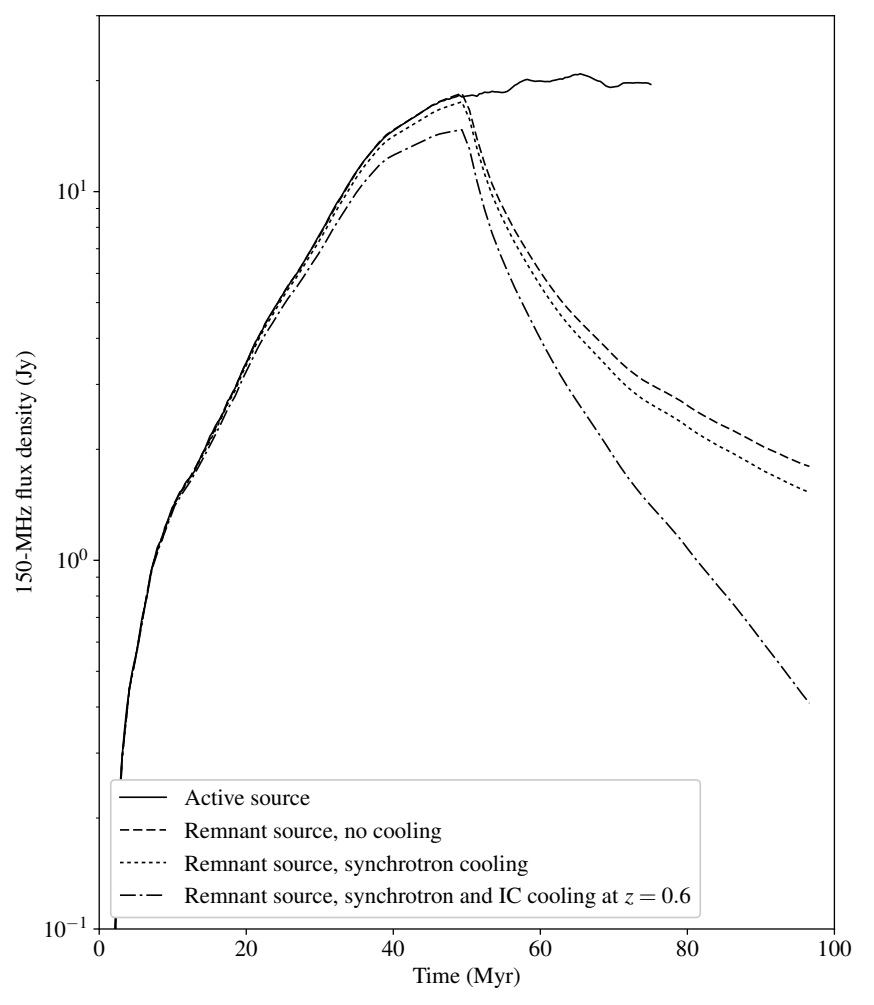

Figure 7. Synchrotron flux density evolutions for the v95-med-m model of Paper 3 without cooling and the r75-60 model without cooling, with only synchrotron cooling and with both synchrotron and inverse-Compton losses assuming the source is at redshift $z=0.6$.

overpressured, having had little time to establish equilibrium with its environment, and would therefore cool faster, the results are actually the other way around: the position within the environment appears to have a greater impact on the amount of time spent in the remnant phase than the stage of the lobes' evolution does.

Comparing the actual values of surface brightness for sources at $z=0.6$ to the LOFAR $3 \sigma$ surface brightness limit, we see that powerful sources like these should remain detectable to a sensitive instrument like LOFAR for a long time. The faintest simulated objects remain an order of magnitude above the LOFAR limit at the end of the simulation, after an elapsed time significantly longer than their active lifetime. Hence, if remnant powerful sources of this type exist, we would expect to find them in LOFAR observations in numbers which would be comparable to or even exceeding those of the parent luminous radio galaxy population. Two caveats are necessary to this statement: firstly, at significantly higher redshift, the effect of inverse-Compton losses would become more severe and would suppress the existence of relics; the second is that, as noted above, the environment of the source and the timing of the termination of jet activity significantly affect the fading timescale. In practice it seems likely that many of these sources will be affected by subsequent restarts of jet activity, a situation recently modelled by Yates, Shabala \& Krause (2018).

Because we have modelled a relatively narrow range in jet power, focussing on the high-power end of the range of realistic radio sources, we cannot directly comment on the remnant fractions seen in LOFAR data as did Mahatma et al. (2018), Godfrey, Morganti \& Brienza (2017) and Hardcastle (2018). We have also considered only a narrow range of shutoff times, as a result of our choice to terminate jet activity when the source reaches $150 \mathrm{kpc}$. As shown by Hardcastle et al. (2019), the properties of low- $z$ radio galaxies are consistent with having a more or less uniform distribution of active times up to $1 \mathrm{Gyr}$, and a more realistic calculation of remnant fraction would require us to simulate jets that switch off over a much wider distribution of lifetimes and of jet powers than is practical in these models. Qualitatively, however, since we find a faster initial drop in the synchrotron luminosity in our simulations than is seen in analytical models, we might expect the true remnant fraction at low $z$ to be lower than the $\sim 50$ per cent of Godfrey, Morganti \& Brienza (2017) or the $\sim 30$ per cent calculated by Hardcastle (2018), and we suggest that a consideration of these effects would bring the models closer to the observations.

Figures 10 and 11 show the decline in surface brightness for our r75-60 model in a more visual form, with Figure 10 showing the synchrotron emission maps from a time-step shortly after the jet is switched off and Figure 11 showing the same model, with the same scaling, at the end of the simulation. We can clearly see the drop in emission across the entire source in the total synchrotron emission maps (top left). Compact structures in both total and polarized intensity have disappeared in the remnant source of Figure 11 and the surface brightness is much more uniform than it was when the source was active. Observers should in principle be able to use these features to help to decide whether a source is a true remnant.

\section{CONCLUSIONS}

We have performed 3D RMHD numerical simulations of radio galaxies in which the jets are shut off, in order to model the remnant phase and test how the observable properties of the remnant depend on the properties of the local cluster environment. In this paper the jets span a narrow range in jet power, one appropriate for powerful FRII radio galaxies, but probe a variety of environments through our choices of the cluster parameters $\beta$ and $r_{c}$. We reproduce the same dynamical growth dependence on environment as seen in Paper 1 and Paper 2 during the active phase, but see that the environments' properties have little effect on the evolution during the remnant phase, with all of the models proceeding to grow at roughly the same rate. In the remnant phase, we find that the fraction of the input energy stored in the shocked shell increases rapidly with time, while the lobes continue to expand out of the cluster centre at speeds significantly higher than the buoyancy speed in most cases. The broad dynamics and energetics of these simulations are similar to those of others in which a jet in a realistic environment is allowed to terminate (Basson \& Alexander 2003; Zanni et al. 2005; Heath, Krause \& Alexander 2007; Perucho et al. 2011; Ito et al. 2015; Hillel \& Soker 2016; Chen, Heinz \& Enßlin 2019) though unlike other authors we focus on the phase immediately after termination.

We find that when the jets are no longer active the cluster gas begins to settle back into the initial potential's distribution and the weight of this infalling gas acts to push some of the dense cluster gas along the jet axis, possibly along channels left by the jet. This dense gas has two major effects on the remnant lobes. Firstly, it acts to push the lobes further out of the cluster, at a faster rate than simply buoyantly rising. As the lobes reach the lower density and pressure regions of the cluster they undergo faster adiabatic losses, and therefore cool faster than would be predicted by models in which the unpowered lobes rise buoyantly out of the cluster. The second effect of this dense cluster gas is to disrupt the structure of the lobes. As the gas gets pushed along the jet axis, it impacts 


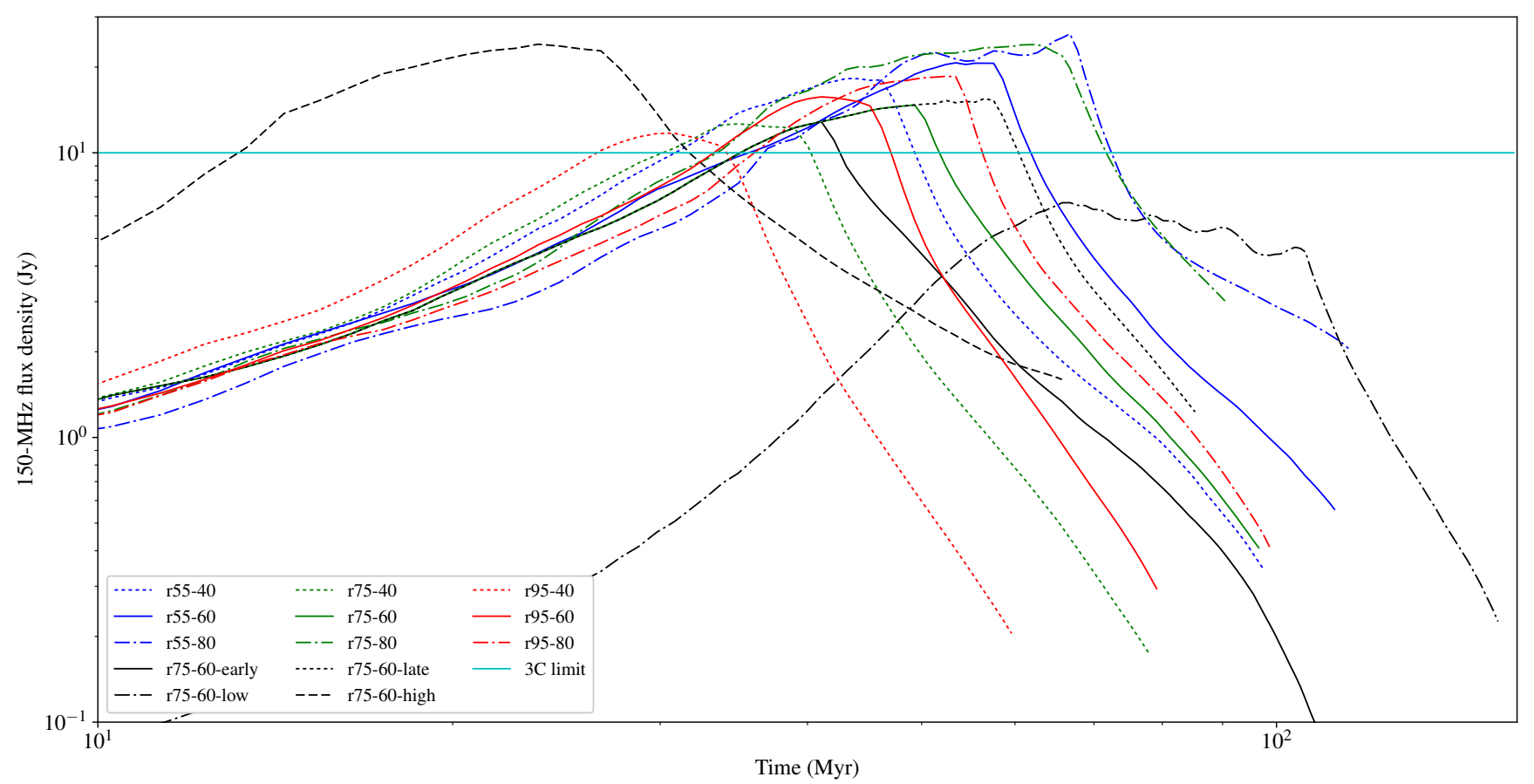

Figure 8. Evolution of the synchrotron flux density with time for the entire suite of simulations, assuming they are at $z=0.6$.

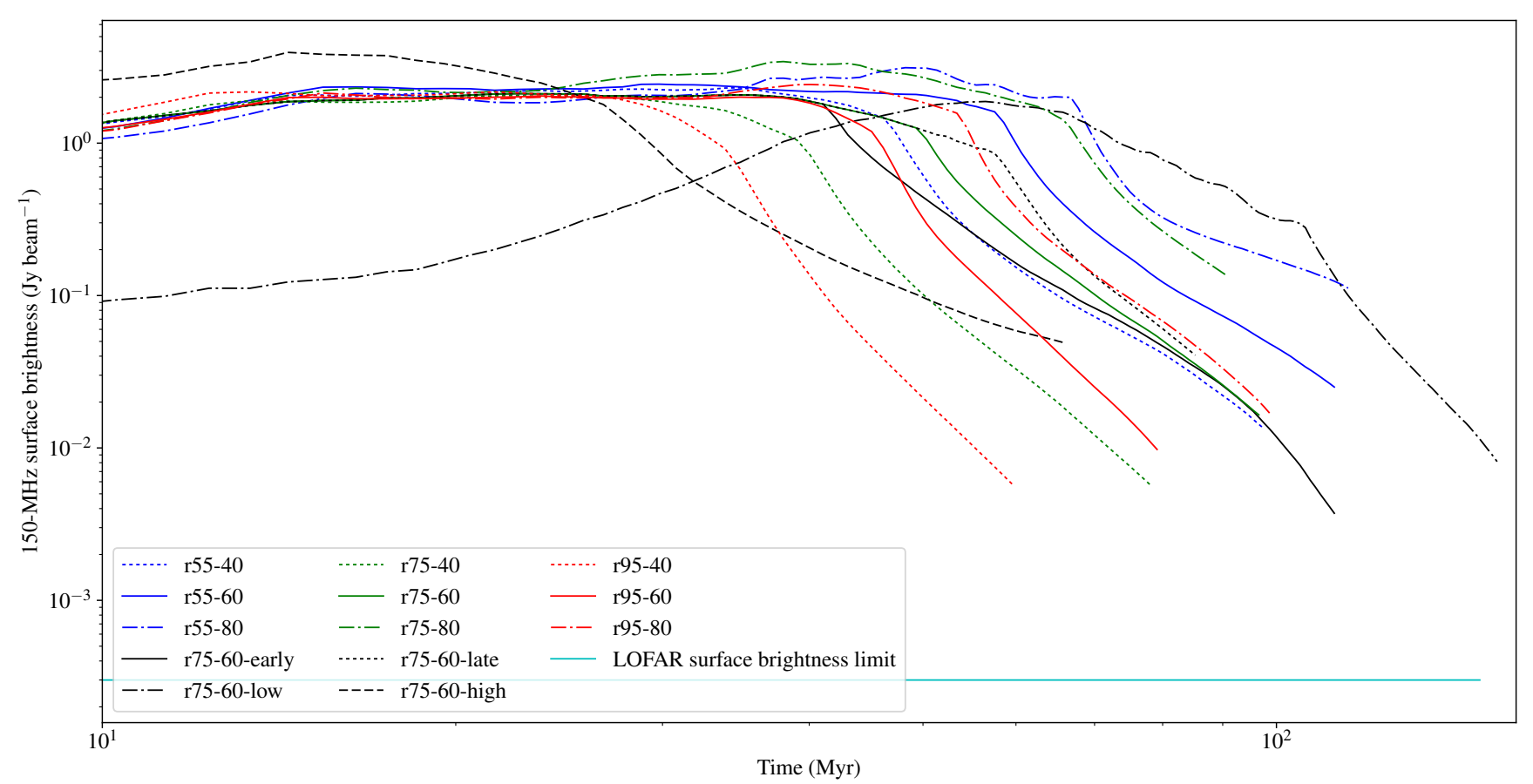

Figure 9. Evolution of the mean surface brightness with time for each model, assuming they are at $z=0.6$ and for a 6 -arcsec Gaussian restoring beam.

the rear of the lobes and begins hollowing them out; eventually, in some models, particularly those with high $\beta$ values, it creates a channel of cluster gas right through to the front of the lobe.

The evolution of surface brightness found in these models has a fairly strong dependence on environmental properties. Both a smaller core radius and a steeper density gradient lead to a significantly shorter time before some threshold in surface brightness is reached, which we attribute to the lobes being embedded in lower pressure cluster material at the time the jets are shut off, resulting in a faster period of adiabatic expansion. Thus, the remnant fraction predicted by simulations is strongly dependent on the environment and the timing of the cessation of jet activity, though in general we expect it to be shorter than it is in analytic models, which do not capture all of the hydrodynamics.

Our models could be improved upon by attempting to solve the issue of the internal boundary coupling well at early times by using models with a higher resolution across the jet. We are limited here by computational power and the two obvious ways around this 


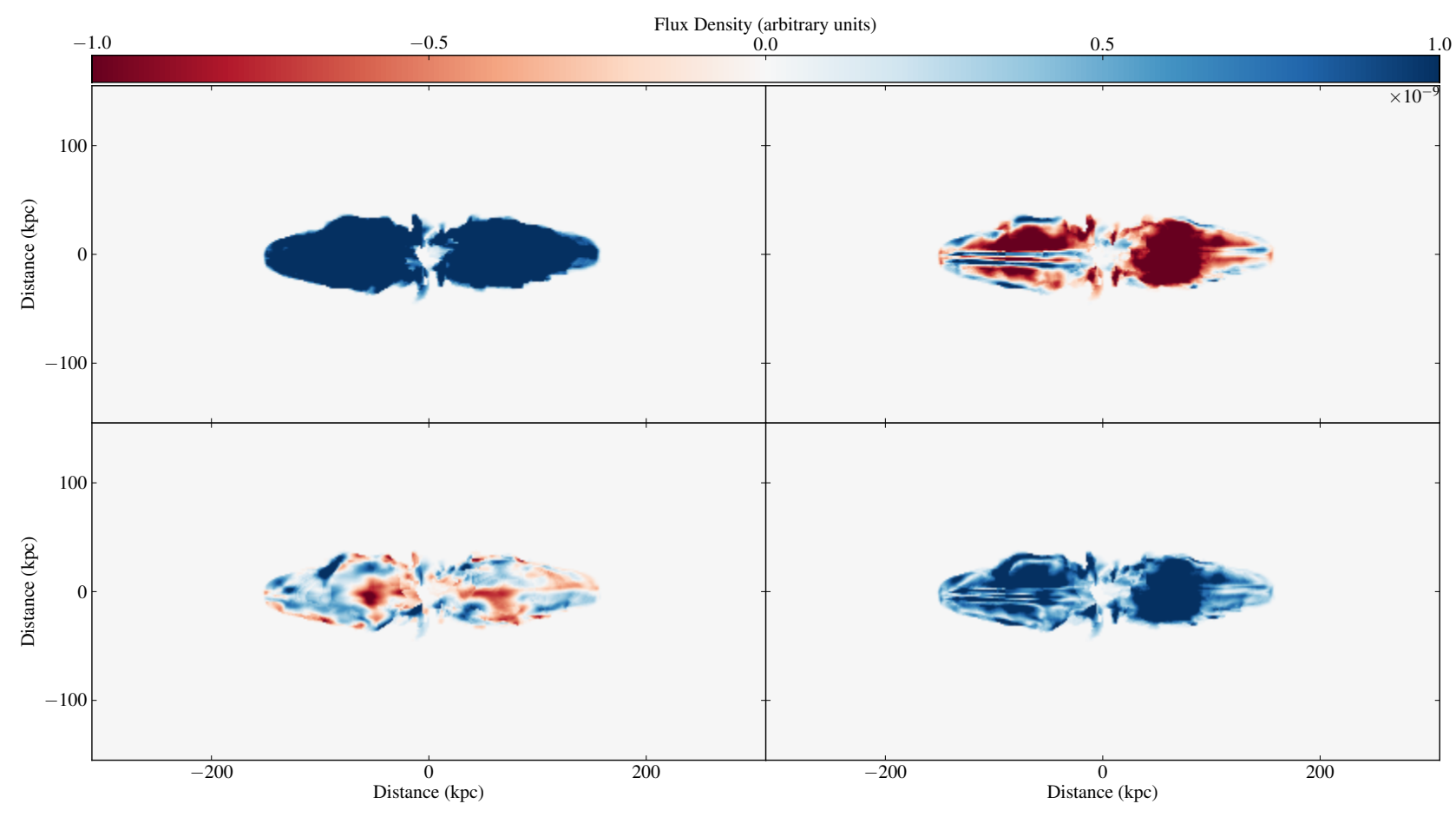

Figure 10. Synchrotron emission maps for the r75-60 simulation, observed at 90 degrees to the jet axis at an age of $51.3 \mathrm{Myr}$, just after the jets have been switched off. Top row: Stokes $I$ (left) and $Q$ (right). Bottom row: Stokes $U$ (left) and $P=\sqrt{Q^{2}+U^{2}}$ (right). All maps are scaled by the same arbitrary amount.

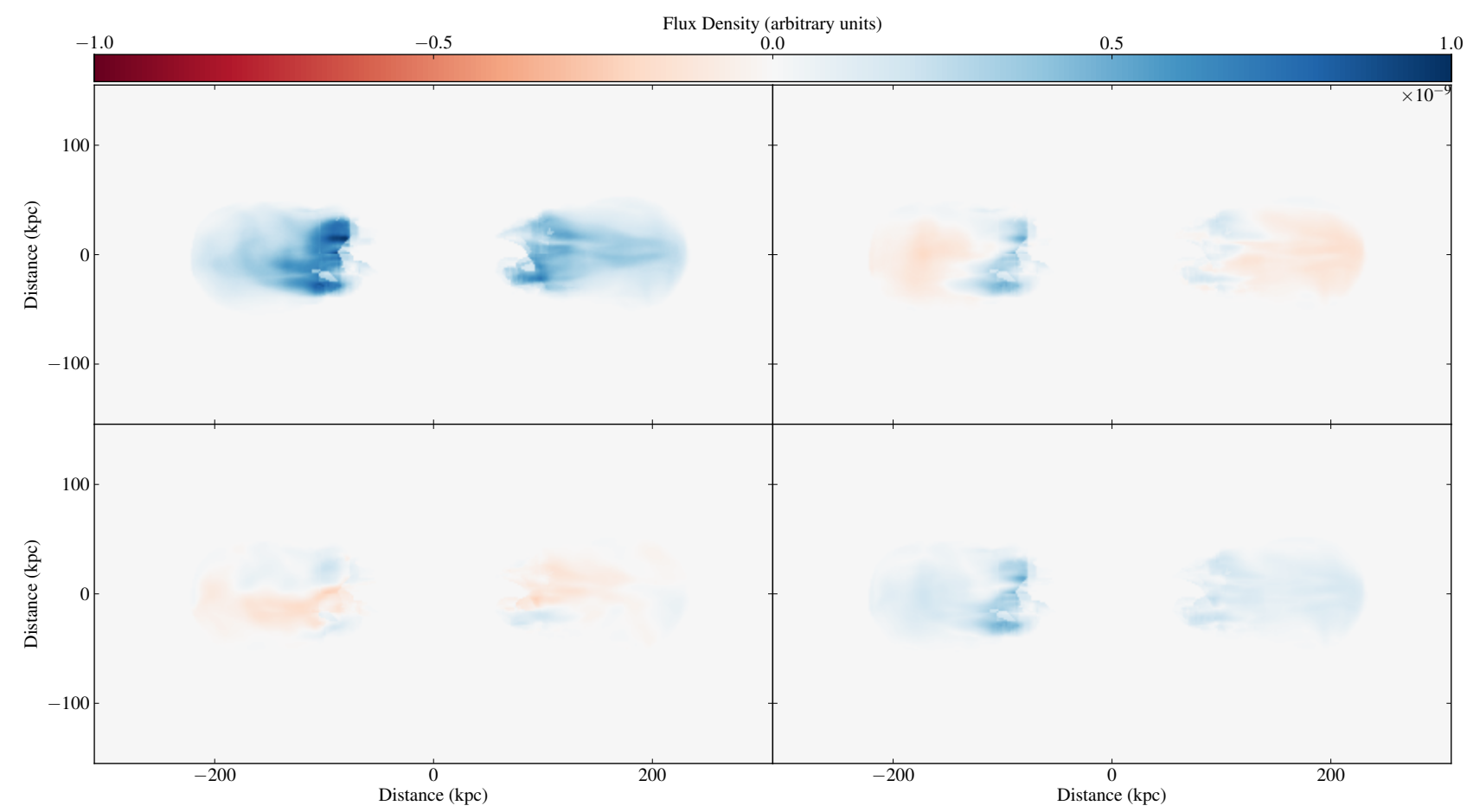

Figure 11. Synchrotron emission maps for the r75-60 simulation, observed at 90 degrees to the jet axis at an age of $92.5 \mathrm{Myr}$, towards the end of the simulation. Top row: Stokes $I$ (left) and $Q$ (right). Bottom row: Stokes $U$ (left) and $P=\sqrt{Q^{2}+U^{2}}$ (right). All maps are scaled by the same arbitrary amount as in Figure 10 so that faint structure can be seen in all of the maps. 
have their own drawbacks. By going back to 2D models we could achieve much greater numerical resolution, but would lose the ability to create realistic synthetic observations. Alternatively, we could make use of PLUTO's adaptive mesh refinement capabilities: however, this is known to affect the small-scale magnetic field structure. Progress is thus likely to require larger volumes in the simulations and more computing power.

Another improvement would be to include a more realistic cooling process, such as the methods of Jones, Ryu \& Engel (1999), where a population of electrons is carried around the grid like tracer quantities in a number of momentum/energy bins. The radiative losses for this population of electrons are then modelled at each time-step and updated, and the resulting population can be used to create much more realistic synthetic observations. Code to implement these models is now under development and will be described in a forthcoming paper.

\section{ACKNOWLEDGMENTS}

We are grateful to the referee, Manel Perucho, for constructive comments that have allowed us to improve the paper.

This work has made use of the University of Hertfordshire high-performance computing facility (https://uhhpc.herts. ac.uk/). WE thanks the UK Science and Technology Facilities Council (STFC) for a studentship [ST/M503514/1]. MJH acknowledges support from STFC through grants [ST/M001008/1] and [ST/R000905/1]. MGHK thanks the University of Tasmania for a Visiting Fellowship.

\section{REFERENCES}

Basson J. F., Alexander P., 2003, MNRAS, 339, 353

Begelman M. C., Blandford R. D., Rees M. J., 1984, Rev. Mod. Phys., 56, 255

Blandford R. D., Rees M. J., 1974, MNRAS, 169, 39

Bourne M. A., Sijacki D., 2017, MNRAS, 472, 4707

Bower R. G., Benson A. J., Malbon R., Helly J. C., Frenk C. S., Baugh C. M., Cole S., Lacey C. G., 2006, MNRAS, 370, 645

Bridle A. H., Perley R. A., 1984, ARA\&A, 22, 319

Brienza M. et al., 2017, A\&A, 606, A98

Chen Y.-H., Heinz S., Enßlin T.A., 2019, MNRAS, 489, 1939

Clarke D. A., Burns J. O., 1991, ApJ 369, 308

Croston J. H., Birkinshaw M., Hardcastle M. J., Worrall D. M., 2004, MNRAS, 353, 879

Croton D. J., et al., 2005, MNRAS, 356, 1155

Cordey R. A., 1986, MNRAS, 219, 575

Cordey R. A., 1987, MNRAS, 227, 695

English W., Hardcastle M. J., Krause M. G. H., 2016, MNRAS, 461, 2025

Gentile G., Rodríguez C., Taylor G. B., Giovannini G., Allen S. W., Lane W. M., Kassim N. E., 2007, ApJ, 659, 225

Giovannini G., Feretti L., Gregorini L., Parma P., 1988, A\&A, 199, 73

Godfrey L. E. H., Morganti R., Brienza M., 2017, MNRAS, 471, 891

Hardcastle M. J., 2013, MNRAS, 433, 3364

Hardcastle M. J., 2018, MNRAS, 475, 2768

Hardcastle M. J., Krause M. G. H., 2013, MNRAS, 430, 174

Hardcastle M. J., Krause M. G. H., 2014, MNRAS, 443, 1482

Hardcastle M. J., Worrall D. M., 2000, MNRAS, 319, 562

Hardcastle M. J., Alexander P., Pooley G. G., Riley J. M., 1997, MNRAS, 288,859

Hardcastle M. J. et al., 2016, MNRAS, 462, 1910

Hardcastle M. J. et al., 2019, A\&A, 622, A12

Heath D., Krause M., Alexander P., 2007, MNRAS, 374, 787

Heinz S., Brüggen M., Young A., Levesque E., 2006, MNRAS, 373, L65
Hillel S., Soker N., 2016, MNRAS, 455, 2139

Ito H., Kino M., Kawakatu N., Orienti M., 2015, ApJ, 806, 241

Jones T. W., Ryu D., Engel A., 1999, ApJ, 512, 105

Kaiser C. R., Cotter G., 2002, MNRAS, 336, 649

Kaiser C. R., Schoenmakers A. P., Röttgering H. J. A., 2000, MNRAS, 315, 381

Konar C., Hardcastle M. J., 2013, MNRAS, 436, 1595

Konar C., Hardcastle M. J., Jamrozy M., Croston J. H., 2013, MNRAS, 430, 2137

Krause M. G. H., 2003, A\&A, 398, 113

Krause M. G. H., 2005, A\&A, 431, 45

Krause M. G. H., Diehl, R., 2014, ApJ, 794, L21

Lind K. R., Payne P. G., Meier D. L., Blandford R. D., 1989, ApJ, 344, 89

Mahatma V. H. et al., 2018, MNRAS, 475, 4557

Mahatma V. H. et al., 2019, A\&A, 622, A13

Mignone A., Bodo G., Massaglia S., Matsakos T., Tesileanu O., Zanni C., Ferrari A., 2007, ApJS, 170, 228

Murgia M., Govoni F., Feretti L., Giovannini G., Dallacasa D., Fanti R., Taylor G. B., Dolag K., 2004, A\&A, 424, 429

Norman M. L., Smarr L., Winkler K.-H. A., Smith M. D., 1982, A\&A, 113, 285

Perucho, M., Quilis, V., Martí, J.M., 2011, ApJ, 743, 42

Perucho, M., Martí, J.M., Quilis, V., Ricciardelli, E., MNRAS, 445, 1462

Schaye J. et al., 2015, MNRAS, 446, 512

Scheuer P. A. G., 1974, MNRAS, 166, 513

Schoenmakers A. P., de Bruyn A. G., Röttgering H. J. A., van der Laan H., Kaiser C. R., 2000, MNRAS, 315, 371

Shimwell T. W., et al., 2017, A\&A, 598, A104

Shimwell T. W., et al., 2019, A\&A, 622, A1

Tchekhovskoy A., Narayan R., McKinney J. C., 2011, MNRAS, 418, L79

Vogelsberger M. et al., 2014, MNRAS, 444, 1518

Yates, P.M., Shabala, S., Krause, M. G. H., 2018, MNRAS, 480, 5286

Zanni C., Murante G., Bodo G., Massaglia S., Rossi P., Ferrari A., 2005, A\&A, 429, 399

This paper has been typeset from a $\mathrm{T}_{\mathrm{E}} \mathrm{X} / \mathrm{L}_{\mathrm{E}} \mathrm{X}$ file prepared by the author. 